\title{
Correction to: Statistical, time series and fractal analysis of full stretch of river Yamuna (India) for water quality management
}

\author{
Rashmi Bhardwaj $^{1}$ - Kulwinder Singh Parmar ${ }^{2}$ \\ Published online: 29 August 2020 \\ (C) Springer-Verlag GmbH Germany, part of Springer Nature 2020
}

Correction to: Environ Sci Pollut Res (2015) 22:397-414 https://doi.org/10.1007/s11356-014-3346-1

As corresponding author, while going through higher research in this area, it is found that the formula for Hurst exponent given in equation (13) on page no. 401 is wrongly written. Accordingly, following corrections suggested:

\section{ABSTRACT}

- $\quad$ On page 397, in Abstract All water quality parameters at every site follows persistent behaviour

instead of

Brownian motion (true random walk) behavior exists at different sites for BOD, AMM, and total Kjeldahl nitrogen $(\mathrm{TKN})$.

- Hurst Exponent is defined as slope of regression line of $\log$-log curve of any time series with time instead of the slope of the regression line. Hurst exponent $(\mathrm{H})$ can be computed on the basis of power law decay i.e.

$\boldsymbol{p}(\boldsymbol{k})=\boldsymbol{C} \boldsymbol{k}^{-\alpha}$ where $C$-constant; $\mathbf{p}(\boldsymbol{k})$

-autocorrelation function with lag $k$

The online version of the original article can be found at https://doi.org/ 10.1007/s11356-014-3346-1

Rashmi Bhardwaj

rashmib@ipu.ac.in; rashmib22@gmail.com

1 University School of Basic \& Applied Sciences (USBAS), Head, Non-Linear-Dynamics-Research-Lab, GGS Indraprastha University, Delhi, India

2 USBAS, GGS Indraprastha University, Delhi, India

Hurst exponent is related to the exponent a by a relation $H=1-\frac{\alpha}{2}$

On page 401

- Delete equation (13)

- Replace $\boldsymbol{p}(\boldsymbol{k})=\boldsymbol{C} \boldsymbol{k}^{-\alpha}$ as Equation (13) instead of equation (14)

- Replace $\boldsymbol{H}=\mathbf{1}-\frac{\alpha}{2}$ as Equation (14) instead of equation (15)

- Replace $\boldsymbol{D}=\mathbf{2}-\boldsymbol{H}$ as Equation (15) instead of equation (16)

- Replace $D(H)=-\lim _{\varepsilon \rightarrow 0} \frac{\ln N(\varepsilon)}{\ln \varepsilon}$ as Equation (16) instead of equation (17)

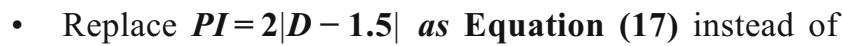
equation (18)

Reference [1]-[3] clearly states the above-mentioned formula. If $0<\mathrm{H}<0.5$, then anti-persistence behavior and if $0.5<\mathrm{H}<1$, then persistence behavior exists. At $\mathrm{H}=0.5$, Brownian motion exists.

$\underline{\text { Results and Discussions: }}$

- On page 401, in Results and Discussions: Table 2 gives regression equation, coefficient of determination of water parameters. Table 3 depicts, Hurst exponent, fractal dimension, and predictability analysis behavior for $S_{1}, S_{2}, S_{3}, S_{4}, S_{5}$. By using equations (1)-(17), the following are observed:

instead of

Table 2 gives regression equation, coefficient of determination, Hurst exponent, fractal dimension, and predictability index, and Table 3 depicts fractal and predictability analysis behavior for $\mathrm{S}_{1}-\mathrm{S}_{2}, \mathrm{~S}_{1}-\mathrm{S}_{3}, \mathrm{~S}_{1}-\mathrm{S}_{4}, \mathrm{~S}_{1}-\mathrm{S}_{5}, \mathrm{~S}_{2}-\mathrm{S}_{3}$, 
$\mathrm{S}_{2}-\mathrm{S}_{4}, \mathrm{~S}_{2}-\mathrm{S}_{5}, \mathrm{~S}_{3}-\mathrm{S}_{4}, \mathrm{~S}_{3}-\mathrm{S}_{5}$, and $\mathrm{S}_{4}-\mathrm{S}_{5}$. By using equations

(1)-(18), the following are observed:

- On page 412, Table 2: Regression equations, coefficient of correlation of water parameters at different sites of River Yamuna

instead of

Table 2 Fractal analysis of water quality parameters

- Also, the values of $\mathbf{H}$ (abs), D (Fractal) and PI from Table 2 to be deleted as Fractal Dimension (D) is based on a single time-series thus, no correlation exists and fractal statistics are calculated for single time-domain only.

- On page 413, Table 3 Fractal Analysis of Water Quality Parameters for Different Sites of Yamuna River (AP- Anti persistence, P- Persistence, B- Brownian time series motion) to be deleted.

- Add Table 3: Fractal Analysis of Water Quality Parameters for Different Sites of Yamuna River. (Table 3 enclosed)

- On page 402, pH: From Table-3, it has been observed that persistent behaviour exists for $\mathrm{pH}$ at all sites $\mathrm{S}_{\mathbf{1}}$, $S_{2}, S_{3}, S_{4}, S_{5}$.

instead of

Anti-persistence behavior exists at all sites except for $\mathrm{S}_{2}-\mathrm{S}_{5}$ which shows persistence behavior.

- On page 404, COD: From Table-3, it has been observed that COD follows persistent behaviour at all sites $S_{1}$, $S_{2}, S_{3}, S_{4}, S_{5}$.

instead of

It is observed that persistence behavior exists for $\mathrm{S}_{1}-\mathrm{S}_{2}, \mathrm{~S}_{1-}$ $\mathrm{S}_{3}$, and $\mathrm{S}_{1}-\mathrm{S}_{4}$; anti-persistence for $\mathrm{S}_{2}-\mathrm{S}_{3}, \mathrm{~S}_{2}-\mathrm{S}_{4}, \mathrm{~S}_{2}-\mathrm{S}_{5}, \mathrm{~S}_{3}-$ $\mathrm{S}_{4}, \mathrm{~S}_{3}-\mathrm{S}_{5}$, and $\mathrm{S}_{4}-\mathrm{S}_{5}$; and Brownian time series (true random walk) for $\mathrm{S}_{1}-\mathrm{S}_{5}$.

- On page 404, BOD: From Table-3, it has been observed that BOD follows persistent behaviour at all sites $S_{1}$, $S_{2}, S_{3}, S_{4}, S_{5}$.

instead of

It is observed that anti-persistence behavior exists for $\mathrm{S}_{2}-\mathrm{S}_{3}$, $\mathrm{S}_{2}-\mathrm{S}_{4}, \mathrm{~S}_{2}-\mathrm{S}_{5}, \mathrm{~S}_{3}-\mathrm{S}_{4}, \mathrm{~S}_{3}-\mathrm{S}_{5}$, and $\mathrm{S}_{4}-\mathrm{S}_{5}$; and Brownian time series (true random walk) for $S_{1}-S_{2}, S_{1}-S_{3}, S_{1}-S_{4}$ and $S_{1}-S_{5}$.

- On page 404, AMM: From Table-3, it has been observed that AMM exhibits persistent behaviour at all sites $S_{1}, S_{2}, S_{3}, S_{4}, S_{5}$. instead of

It is observed that persistence behavior exists for $\mathrm{S}_{1}-\mathrm{S}_{5}, \mathrm{~S}_{3}-$ $\mathrm{S}_{5}$, and $\mathrm{S}_{4}-\mathrm{S}_{5}$; anti-persistence for $\mathrm{S}_{2}-\mathrm{S}_{3}, \mathrm{~S}_{2}-\mathrm{S}_{4}, \mathrm{~S}_{2}-\mathrm{S}_{5}$ and $\mathrm{S}_{3}-\mathrm{S}_{4}$; and Brownian time series (true random walk) for $\mathrm{S}_{1^{-}}$ $\mathrm{S}_{2}, \mathrm{~S}_{1}-\mathrm{S}_{3}$ and $\mathrm{S}_{1}-\mathrm{S}_{4}$.

- On page 404 and 407, TKN: From Table-3, it has been observed that AMM exhibits persistent behaviour at all sites $S_{1}, S_{2}, S_{3}, S_{4}, S_{5}$.

instead of

It is observed that persistence behavior exists for $\mathrm{S}_{1}-\mathrm{S}_{5}$; antipersistence for $\mathrm{S}_{2}-\mathrm{S}_{3}, \mathrm{~S}_{2}-\mathrm{S}_{4}, \mathrm{~S}_{2}-\mathrm{S}_{5}, \mathrm{~S}_{3}-\mathrm{S}_{4}, \mathrm{~S}_{3}-\mathrm{S}_{5}$ and $\mathrm{S}_{4}-\mathrm{S}_{5}$ and Brownian time series (true random walk) for $\mathrm{S}_{1}-\mathrm{S}_{2}, \mathrm{~S}_{1}-\mathrm{S}_{3}$ and $\mathrm{S}_{1}-\mathrm{S}_{4}$.

- On page 408 and 409, DO: From Table-3, it has been observed that DO observes persistent behaviour at all sites $S_{1}, S_{2}, S_{3}, S_{4}, S_{5}$.

instead of

It is observed that persistence behavior exists for $\mathrm{S}_{1}-\mathrm{S}_{2}, \mathrm{~S}_{1}-$ $\mathrm{S}_{3}, \mathrm{~S}_{1}-\mathrm{S}_{4}, \mathrm{~S}_{2}-\mathrm{S}_{5}$ and $\mathrm{S}_{3}-\mathrm{S}_{5}$; anti-persistence for $\mathrm{S}_{1}-\mathrm{S}_{5}, \mathrm{~S}_{2}-\mathrm{S}_{3}$, $\mathrm{S}_{2}-\mathrm{S}_{4}, \mathrm{~S}_{3}-\mathrm{S}_{4}$ and $\mathrm{S}_{4}-\mathrm{S}_{5}$.

- On page 410 and 411, WT: From Table-3, it has been observed that persistent behaviour exists for WT at all sites $S_{1}, S_{2}, S_{3}, S_{4}, S_{5}$.

instead of

It is observed that anti-persistence behavior exists for $\mathrm{S}_{1}-\mathrm{S}_{2}$, $\mathrm{S}_{1}-\mathrm{S}_{3}, \mathrm{~S}_{1}-\mathrm{S}_{4}, \mathrm{~S}_{1}-\mathrm{S}_{5}, \mathrm{~S}_{2}-\mathrm{S}_{3}, \mathrm{~S}_{2}-\mathrm{S}_{4}, \mathrm{~S}_{2}-\mathrm{S}_{5}, \mathrm{~S}_{3}-\mathrm{S}_{4}, \mathrm{~S}_{3}-\mathrm{S}_{5}$ and $\mathrm{S}_{4}-\mathrm{S}_{5}$.

Conclusion:

- Also, on page 413 in Conclusion, Persistent Behaviour exists for all water quality parameters at all sites of Yamuna river; therefore long term behaviour of water quality trend is predictable.

instead of

Brownian motion (true random walk) behavior exists at different sites for BOD, AMM, and TKN; therefore, water quality trend is unpredictable.

\section{References}

1. Munshi, J (2019) There is no chaos in stock markets (June 11, 2014). Available at SSRN: https://ssrn.com/ abstract $=2448648$ or https://doi.org/10.2139/ssrn. 2448648

2. Watkins, NW (2019) Mandelbrot's stochastic time series models. Earth Space Sci. 6:2044-2056. https://doi.org/10. 1029/2019EA000598 
Table 3 Fractal analysis of water quality parameters for different sites of Yamuna River

\begin{tabular}{|c|c|c|c|c|c|}
\hline Water Parameters & Sample sites & Hurst Exponent & Fractal Dimension & Predictability Index (PI) & Behaviour \\
\hline \multirow[t]{5}{*}{$\mathrm{pH}$} & Hathnikund $\left(\mathrm{S}_{1}\right)$ & 0.6583 & 1.3417 & 0.3166 & Persistent \\
\hline & Nizamuddin $\left(\mathrm{S}_{2}\right)$ & 0.6657 & 1.3343 & 0.3314 & Persistent \\
\hline & Mazawali $\left(\mathrm{S}_{3}\right)$ & 0.5998 & 1.4002 & 0.1996 & Persistent \\
\hline & Agra DS $\left(\mathrm{S}_{4}\right)$ & 0.5934 & 1.4066 & 0.1868 & Persistent \\
\hline & Juhika $\left(\mathrm{S}_{5}\right)$ & 0.6681 & 1.3319 & 0.3362 & Persistent \\
\hline \multirow[t]{5}{*}{ COD } & Hathnikund $\left(\mathrm{S}_{1}\right)$ & 0.5801 & 1.4199 & 0.1602 & Persistent \\
\hline & Nizamuddin $\left(\mathrm{S}_{2}\right)$ & 0.5763 & 1.4237 & 0.1526 & Persistent \\
\hline & Mazawali $\left(\mathrm{S}_{3}\right)$ & 0.6108 & 1.3892 & 0.2216 & Persistent \\
\hline & Agra DS $\left(\mathrm{S}_{4}\right)$ & 0.6604 & 1.3396 & 0.3208 & Persistent \\
\hline & Juhika $\left(\mathrm{S}_{5}\right)$ & 0.5681 & 1.4319 & 0.1362 & Persistent \\
\hline \multirow[t]{5}{*}{ BOD } & Hathnikund $\left(\mathrm{S}_{1}\right)$ & 0.6441 & 1.3559 & 0.2882 & Persistent \\
\hline & Nizamuddin $\left(\mathrm{S}_{2}\right)$ & 0.6093 & 1.3907 & 0.2186 & Persistent \\
\hline & Mazawali $\left(\mathrm{S}_{3}\right)$ & 0.5982 & 1.4018 & 0.1964 & Persistent \\
\hline & Agra DS $\left(\mathrm{S}_{4}\right)$ & 0.6486 & 1.3514 & 0.2972 & Persistent \\
\hline & Juhika $\left(\mathrm{S}_{5}\right)$ & 0.6210 & 1.379 & 0.242 & Persistent \\
\hline \multirow[t]{5}{*}{ AMM } & Hathnikund $\left(\mathrm{S}_{1}\right)$ & 0.6349 & 1.3651 & 0.2698 & Persistent \\
\hline & Nizamuddin $\left(\mathrm{S}_{2}\right)$ & 0.6316 & 1.3684 & 0.2632 & Persistent \\
\hline & Mazawali $\left(\mathrm{S}_{3}\right)$ & 0.6471 & 1.3529 & 0.2942 & Persistent \\
\hline & Agra DS $\left(\mathrm{S}_{4}\right)$ & 0.6346 & 1.3654 & 0.2692 & Persistent \\
\hline & Juhika $\left(\mathrm{S}_{5}\right)$ & 0.6064 & 1.3936 & 0.2128 & Persistent \\
\hline \multirow[t]{5}{*}{ TKN } & Hathnikund $\left(\mathrm{S}_{1}\right)$ & 0.6436 & 1.3564 & 0.2872 & Persistent \\
\hline & Nizamuddin $\left(\mathrm{S}_{2}\right)$ & 0.6102 & 1.3898 & 0.2204 & Persistent \\
\hline & Mazawali $\left(\mathrm{S}_{3}\right)$ & 0.6497 & 1.3503 & 0.2994 & Persistent \\
\hline & Agra DS $\left(\mathrm{S}_{4}\right)$ & 0.6512 & 1.3488 & 1.6512 & Persistent \\
\hline & Juhika $\left(\mathrm{S}_{5}\right)$ & 0.6329 & 1.3671 & 0.2658 & Persistent \\
\hline \multirow[t]{5}{*}{ DO } & Hathnikund $\left(\mathrm{S}_{1}\right)$ & 0.6127 & 1.3873 & 0.2254 & Persistent \\
\hline & Nizamuddin $\left(\mathrm{S}_{2}\right)$ & 0.5643 & 1.4357 & 0.1286 & Persistent \\
\hline & Mazawali $\left(\mathrm{S}_{3}\right)$ & 0.5955 & 1.4045 & 0.191 & Persistent \\
\hline & Agra DS $\left(\mathrm{S}_{4}\right)$ & 0.5775 & 1.4225 & 0.155 & Persistent \\
\hline & Juhika $\left(\mathrm{S}_{5}\right)$ & 0.5831 & 1.4169 & 0.1662 & Persistent \\
\hline \multirow[t]{5}{*}{ WT } & Hathnikund $\left(\mathrm{S}_{1}\right)$ & 0.5520 & 1.448 & 0.104 & Persistent \\
\hline & Nizamuddin $\left(\mathrm{S}_{2}\right)$ & 0.5580 & 1.442 & 0.116 & Persistent \\
\hline & Mazawali $\left(\mathrm{S}_{3}\right)$ & 0.5631 & 1.4369 & 0.1262 & Persistent \\
\hline & Agra DS $\left(\mathrm{S}_{4}\right)$ & 0.5724 & 1.4276 & 0.1448 & Persistent \\
\hline & Juhika $\left(\mathrm{S}_{5}\right)$ & 0.5426 & 1.4574 & 0.0852 & Persistent \\
\hline
\end{tabular}

3. Kirichenko L, Radivilova T, Bulakh V (2019) Machine learning in classification time series with fractal properties. Data 2019, 4:5. https://doi.org/10.3390/data4010005

In test add Table 3

Publisher's note Springer Nature remains neutral with regard to jurisdictional claims in published maps and institutional affiliations. 